\title{
Considerations regarding the new requirements for the evaluation of rotating electric machines with increased safety protection type "e"
}

\author{
Marcel Daniel $\operatorname{Rad}^{l, *}$, Diana Sălăsan ${ }^{l}$, Sorin Zsido ${ }^{l}$ \\ ${ }^{1}$ National Institute for Research and Development in Mine Safety and Protection to Explosion - \\ INSEMEX PETROSANI -Romania
}

\begin{abstract}
The evaluation of explosion-proof electrical equipment for certification is particularly important given the risk of explosion and must be minimized. This in order to ensure the safety of life, health of workers, to prevent damage to goods and the environment when they meet the essential security requirements at European level. Directive 2014/34 / EU states that equipment used in explosive atmospheres must be designed to operate without endangering the environment for which it is intende. This paper proposes the study and evaluation of the requirements for the evaluation of rotating electric machines with increased safety protection type "e". The rotating electric machines with increased safety protection type "e", in order to comply with the explosion protection, it must meet certain requirements given by the standard SR-EN 60079-7.
\end{abstract}

\section{Introduction}

Generally speaking, designing and manufacturing of electrical apparatus benefits lately of special advantages, offered by the appearance of new components (integrated) and technologies, which makes the time that passes from enouncing the idea until physical achievement of the apparatus to be relatively short, and the process involved by that to be a monotonous one.

But, when the problem of adapting this apparatus to the particularities of use them in atmospheres with explosion hazard, the above mentioned process is considerably slowed, not by the missing of consecrated components for such processes, but especially by the leak of experience and knowledge regarding the standard requirements, referring to construction and using of electrical apparatus in areas with hazard of explosive atmosphere.[2]

This state of fact is negatively more emphatic because, lately, the groups of standards from this field in the world, Europe and Romania have a peculiar dynamic caused especially by the homogenisation and generalisation process opened and maintained by SR-EN.

Evaluation and testing of equipments that compose an explosion-proof system, in purpose of certification, is very important considering the existing explosion risk which has to be minimized to ensure peoples health and security, as well as to prevent goods damage and, not in the last instance, to protect the environment.

*Corresponding author: marcel.rad@insemex.ro 
Electrical equipment and Ex Components of type of protection increased safety " $\mathrm{e}$ " are either:

a) Level of Protection "eb" (EPL "Mb" or "Gb"); or

b) Level of Protection "ec" (EPL "Gc")

The type of protection increased safety "e" applies to electrical apparatus with a rated value of supply voltage not exceeding $11 \mathrm{kV}$ r.m.s. a.c. or d.c.

For Level of Protection "ec", this standard applies to electrical equipment where the rated voltage does not exceed $15 \mathrm{kV}$ r.m.s., a.c. or d.c.[3]

Level of Protection "eb" applies to equipment or Ex Components, including their connections, conductors, windings, lamps, and batteries; but not including semiconductors or electrolytic capacitors.

Level of Protection "ec" applies to equipment or Ex Components, including their connections, conductors, windings, lamps, and batteries; and also including semiconductors and electrolytic capacitors.[4]

Additional measures are applied to ensure that the apparatus does not produce arcs, sparks, or excessive temperatures in normal operation or under specified abnormal conditions.

The principle for the type of protection increased safety is represented by carefully choose of the materials used for construction of such kind of apparatus, assurance of certain clearances and creepage distances in such manner that the probability of a failure to occur and to result an electric arc or spark to be reduced at an acceptable level; as well as to ensure an adequate degree of protection for the apparatus enclosure. [4]

For the certification of electrical apparatus with type of protection increased safety, this should be submitted to type tests and routine tests.

In the type tests category are included the tests to determine the maximum surface temperature (to include the apparatus in a certain temperature class), tests for resistance to impact, dielectric strength test, tests for degree of protection (IP) and, if necessary, tests for thermal endurance to heat and cold, resistance to light, resistance to chemical agents and other tests which are specific for different types of apparatus.[3]

\section{Classification of explosive atmospheres. Hazard of explosion}

To have an explosion three factors must exist at the same time and in the same space. These factors form the triangle of explosion hazard (fig. 1):

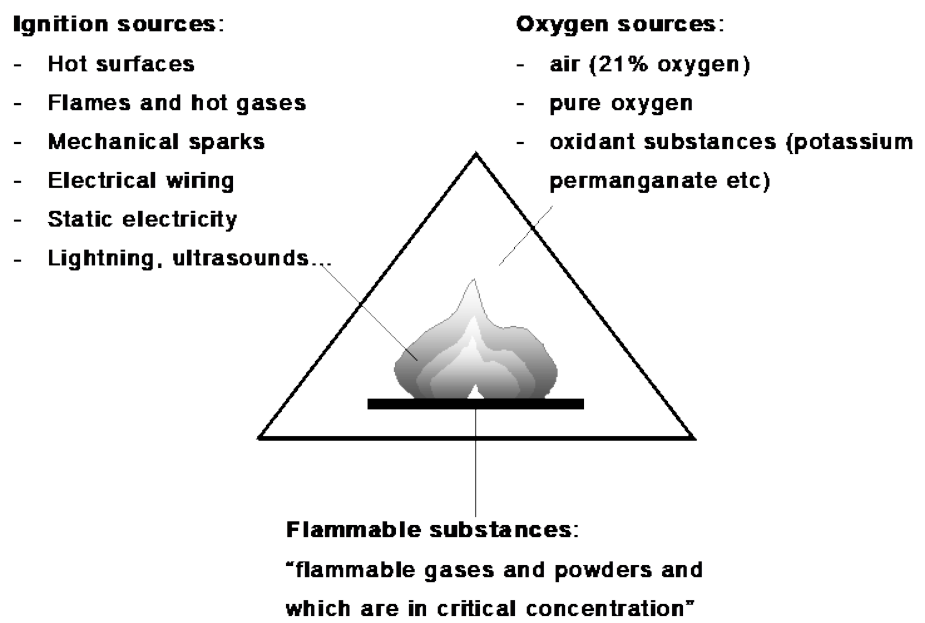

Fig. 1. Ignition triangle 
Presence of flammable substances in form of gases, vapours, mists;

Presence of oxidant substance, air or oxygen, as support for violent combustion (explosion);

Presence of ignition source in form of sparks and hot surfaces.[5]

\section{Types of protection - short description}

The type of protection represents a technical solution by which at least one of the factors represented in the ignition triangle is removed or limited below the critical values.

Flameproof enclosure

Represents a type of protection that permits to have an explosion inside equipment, but which by the characteristic elements for this type of protection (flameproof joints) makes that explosion not to be transmitted to the explosive atmosphere that surrounds the enclosure. Generally, it is used for power apparatus, but can also be used for other kinds of apparatus. [5]

Increased safety

A type of protection which consists in applying some supplementary measures to avoid producing electric arcs, sparks, or excessive temperatures on any part of electrical apparatus (internal or external). These phenomena are not produced even in normal operation.[5]

Non-incendive

Represents a type of protection which is based on the other types of protection principles, but it contains less rigorous prescriptions than those contained in the standards for types of protection eligible in zone 1 . This type of protection is only eligible for zone 2. [5]

Intrinsic safety

Represents a type of protection by which electrical parameters are safely limited so than the ignition source to be limited to a non hazardous value. This is also a consecrated type of protection for "low currents" applications.[5]

Encapsulation

It is a type of protection by which the small kind apparatus is separated from hazardous atmosphere by moulding / enclosing in compound. [5]

Pressurization

It's a type of protection by which the apparatus (often) in normal construction is placed inside an enclosure in which a protective gas is circulated so as in the inner space the explosive gas concentration is much lower than the lower explosive limit (LEL). Pressurization remains the only available solution for high frame sizes apparatus.[5]

\section{Rotating electric machines with increased safety protection type "e"}

The new requirements for rotating electric machines with the type of increased safety protection "e" given by the SR-EN 60079-7 / 2016 refer to the assessment for possible air gap sparking, limiting temperature of rotor, limiting temperature by current-dependent safety device, limiting temperature by temperature and other sensors.

Assessment for possible air gap sparking

Rotating electrical machines shall be assessed for possible air gap sparking as follows. For Level of Protection "ec" this assessment shall only be required for machines with a rated output exceeding $100 \mathrm{~kW}$ with duty types S3, S4, S5, S7, S8, or S10. If the total sum of the factors determined by SR-EN 60079-7_is greater than 6, one of the following additional protective measures shall be applied: 
a) The machine or a representative sample shall be tested in accordance with SR-EN 60079-7 2016

b) The machine design shall allow special measures to be applied during starting, to ensure

That its enclosure does not contain an explosive gas atmosphere at the time of starting. In this case, the certificate number shall include the " $X$ " suffix in accordance with the marking requirements of SR-EN 60079-0 and the Specific Conditions of Use listed on the certificate shall detail the special measures to be applied.[1] $\mathrm{I}_{\mathrm{N}}$

c) The starting current of the machine is required to be limited to $300 \%$ of rated current,

When external current limiting is required, the certificate number shall include the " $X$ " suffix in accordance with the marking requirements of SR-EN 60079-0 and the Specific Conditions of Use listed on the certificate shall detail that the motor is suitable only for reduced voltage starting which limits the starting current to $300 \%$ of the rated current.

Limiting temperature of rotor.

For rotating electrical machines with cage rotors, including synchronous machines with 'cage rotor' starting or damping windings, the limiting temperature of the rotor shall not exceed $300{ }^{\circ} \mathrm{C}$ for uninsulated rotor cage constructions or the temperature limitation_for insulated rotor cage constructions. For Level of Protection "eb" motors of duty types S1 to S10 and Level of Protection "ec" motors of duty types S3, S4, S5, S7, S8, or S10, the limiting temperature of the rotor shall not be exceeded, even under starting conditions.

Limiting temperature by current-dependent safety device

For rotating machines of Level of Protection "eb" intended for line starting protected by a current-dependent safety device to protect against exceeding the limiting temperature, the starting current ratio $\mathrm{I}_{\mathrm{A}} / \mathrm{I}_{\mathrm{N}}$ and the time $\mathrm{t}_{\mathrm{E}}$ shall be determined and marked in accordance with SR-EN 60079-7 2016.[1]

The length of time $t_{E}$ shall be such that, when the machine is stalled, it can be disconnected by the current-dependent safety device before time $t_{\mathrm{E}}$ has elapsed. In general, this is possible if the minimum values for $t_{E}$ given in Fig. 2. as a function of the starting current ratio $I_{A} / I_{N}$ are exceeded. Values of time t below the values in Fig. 2. are permissible only if a suitable current-dependent safety device is used for the machine and it is shown to be effective by test. The current-dependent safety devices shall be specified by marking on the machine in accordance with SR-EN 60079-7 2016.[1]

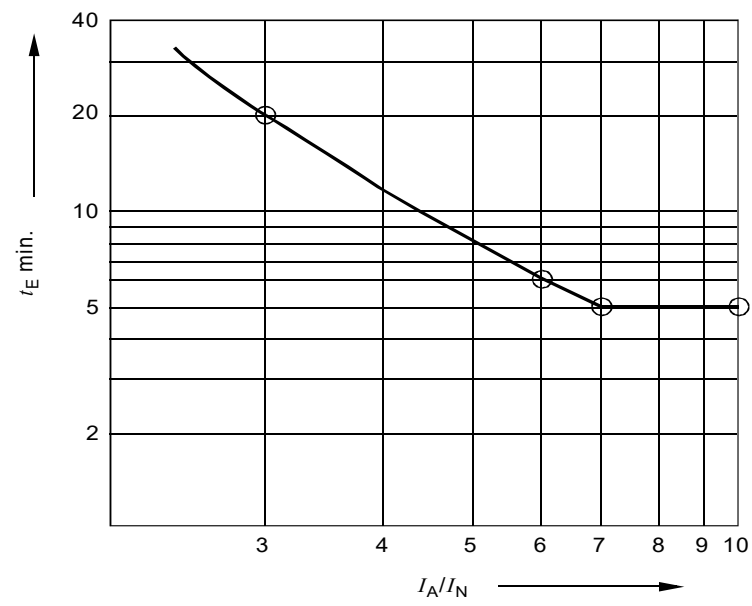

Fig. 2. Minimum values of the time $t$ (in seconds) of motors in relation to the starting current ratio $I_{A} /$ $\mathrm{IN}_{\mathrm{N}}$ 
In no case,

a) shall the value of time $t_{E}$ be less than $5 \mathrm{~s}$ when using a current-dependent safety device,

b) shall the value of the starting current ratio $\mathrm{I}_{\mathrm{A}} / \mathrm{I}_{\mathrm{N}}$ exceed 10 .

Thermal protection of Level of Protection "ec" machines during expected malfunctions is provided by the general industrial requirements and no additional safety devices are necessary to maintain the explosion protection.

Limiting temperature by temperature and other sensors.

Even for rotating machines of Level of Protection "eb" intended for use with winding temperature sensors associated with safety devices to protect against exceeding the limiting temperatures, the starting current ratio $\mathrm{I}_{\mathrm{A}} / \mathrm{I}_{\mathrm{N}}$ shall be determined and marked in accordance with_SR-EN 60079-7 2016. Time $t_{\mathrm{E}}$ is not required to be determined and marked.

For polyphase machines, there shall be a minimum of one sensor per phase.

Winding temperature sensors associated with safety devices shall be considered adequate for the thermal protection of the machine if the requirements of 4,8 are satisfied even with the rotor locked, other than duty types S1, S2, S6, or S9 in Level of Protection "ec". The requirements for associated safety devices shall be specified by marking on the machine in accordance with SR-EN 60079-7 2016.

In no case shall the value of the starting current ratio $\mathrm{I}_{\mathrm{A}} / \mathrm{I}_{\mathrm{N}}$ be more than 10 .

Speed sensors, impedance relays, rate-of-rise sensors, etc. along with associated safety devices may also be used to limit the rotor temperature.

For machines that are rotor limited, it is not generally practical to limit the rotor temperature by the use of stator winding temperature sensors alone. Additional considerations such as different rates of rise and selection of the tripping point of the sensor might be relevant.

Thermal protection of Level of Protection "ec" machines during expected malfunctions is provided by the general industrial requirements and no additional safety devices are necessary to maintain the explosion protection.[1]

\section{Conclusions}

The purpose of the paper is to underline the new tests that can be done at INCD INSEMEX Petrosani for electrical apparatus with increased safety „e" type of protection. These tests are: thermal endurance to heat and cold, determination of surface temperature for electric motors and determination of time $t_{\mathrm{E}}$ for electrical rotating machines.

\section{References}

1. Standard SR EN 60079-7 (2016)

2. Directive 2014/34/EU (2014)

3. L. Moldovan, S. Burian, G.A. Gaman, M. Paraian, M. Magyari, Proceedings of the 17th International Multidisciplinary Scientific Geoconference SGEM 2017 17, 531-538 (2017)

4. M. D. Rad, M. Magyari, L. Moldovan, D. Fotau, D. Salasan, Proceedings of the 20th International Multidisciplinary Scientific Geoconference SGEM 2020 20, 657-664 (2020)

5. Standard SR EN 60079-0 (2013) 